\title{
Estimación de la pérdida de colonias de abejas melíferas en el altiplano y el norte de México
}

\section{Estimation of the loss of honey bee colonies in the altiplano and northern regions of Mexico}

\author{
Carlos Aurelio Medina-Flores ${ }^{1}$, Nancy Harlet Esquivel-Marín ${ }^{1}$, Marco López-Carlos ${ }^{1}$, Sergio Ernesto \\ Medina-Cuellar ${ }^{2}$, Jairo Iván Aguilera-Soto ${ }^{1 *}$
${ }^{1}$ Unidad Académica de Medicina Veterinaria y Zootecnia, Universidad Autónoma de Zacatecas. Carretera Panamericana Zacatecas-Fresnillo km 31.5, El Cordovel, Enrique Estrada. CP. 98500, Zacatecas, México. Santiago km 3.5+1.8, CP. 36885, Salamanca, Guanajuato, México
*Autor de correspondencia: aguileraivan@yahoo.com.mx \\ ${ }^{2}$ Universidad de Guanajuato campus Irapuato-Salamanca, Departamento de Arte y Empresa, Carretera Salamanca-Valle de
}

Nota científica recibida: 10 de mayo de 2017 aceptada: 18 de octubre de 2017

RESUMEN. El objetivo fue estimar la tasa de pérdida de colonias de abejas melíferas (Apis mellifera) durante el invierno de 2015-2016 en las regiones del altiplano y norte de México. Se encuestaron 196 apicultores ( $n=41907$ colonias). La tasa promedio de pérdida de colonias en ambas regiones fue del 33.4\%, no se presentaron diferencias significativas $(p>0.05)$ entre regiones. Los apicultores encuestados estiman que las principales causas de pérdida fueron: el mal tiempo, enfermedades, insecticidas, evasión y la enjambrazón. No hubo efecto del tamaño de las explotaciones apícolas respecto a la tasa de pérdida de colonias. El presente estudio representa la primer estimación en dos regiones de México sobre la tasa de pérdida de colonias en el periodo invernal y que ésta es similar a la de otros países. Se sugiere implementar técnicas de manejo que contribuyan a mitigar los efectos del mal clima, las enfermedades, plagas y la africanización.

Palabras clave: Apis mellifera, encuesta, mortalidad, síndrome del colapso de la colonia, Varroa destructor

ABSTRACT. The objective was to estimate the rate of loss of honey bee (Apis mellifera) colonies during the winter of 2015-2016 in the altiplano and northern regions of Mexico. In total, 196 beekeepers ( $n=41907$ colonies) were surveyed. The average rate of colony loss in both regions was $33.4 \%$, with no significant differences $(p>0.05)$ between regions. Beekeepers surveyed estimate that the main causes of loss were: bad weather, diseases, insecticides, evasion and swarming. The size of apicultural farms had no effect on the rate of colony loss. This study represents the first estimate in two regions of Mexico of the rate of colony loss in the winter period, which is similar to that of other countries. It is suggested to implement management techniques that would help mitigate the effects of bad weather, diseases, pests and Africanization.

Key words: Apis mellifera, survey, mortality, colony collapse syndrome, Varroa destructor

\section{INTRODUCCIÓN}

Actualmente, la salud de los ecosistemas es amenazada por la pérdida masiva de colonias de abejas melíferas, principalmente en países del hemisferio norte (Neuman y Carreck 2010). Durante los últimos diez años, los apicultores de Canadá, EUA y varios países europeos han registrado pérdidas anuales de alrededor del $30 \%$ (Oldroyd 2007, Van Engelsdorp 2008, 2010, Chauzat et al. 2010, Guzmán-Novoa et al. 2010), y hasta del $90 \%$ de sus colonias (Stankus 2014), esto ha provocado desabasto de polinizadores para los cultivos (Guzmán-Novoa 2016). Dicho problema se conoce como síndrome del colapso de la colonia (Van Engelsdorp et al. 2009), el cual no solo 
es inusual por haber una elevada mortalidad, sino porque no existe una causa específica, debido a que por lo general no hay cadáveres, se presenta una despoblación súbita con desproporción cría-abejas (Oldroyd 2007).

Aun cuando la razón de las elevadas tasas de mortalidad no es completamente conocida, se ha reportado que el problema se debe al efecto $e$ interacción de factores como plagas y enfermedades, estrés de la colonia provocado por prácticas apícolas intensivas como la movilización de colmenas, deficiente alimentación a causa del clima o por la exposición de las colonias a una sola fuente floral durante la polinización, además de inclemencias ambientales y pesticidas (Van Engelsdorp et al. 2009, GuzmánNovoa et al. 2010, Stankus 2014). El efecto aditivo de estos factores puede inhibir la respuesta inmune de las abejas, haciéndolas más susceptibles a colapsar (Guzmán-Novoa 2016).

La determinación de la tasa de pérdidas de colonias de abejas es fundamental para situar al problema en su justa dimensión e identificar posibles causas de mortalidad, para esto, la aplicación de encuestas a apicultores ha representado una importante alternativa (Lee et al. 2015). Por lo anterior, el objetivo del presente estudio fue estimar la tasa de pérdida de colonias durante el invierno de 20152016, así como de los factores asociados, mediante la aplicación de una encuesta a apicultores de dos regiones apícolas de México.

\section{MATERIALES Y MÉTODOS}

Se encuestó vía telefónica, por medios electrónicos (página web, correo electrónico y redes sociales) y de forma personal a 196 apicultores de 14 estados de la república mexicana, que representan a dos regiones apícolas del país (CGG 2010). En la región norte se encuestaron 110 apicultores de los estados de Coahuila, Chihuahua, Durango y Zacatecas, mientras que en la región del altiplano se encuestaron 86 apicultores de Aguascalientes, Ciudad de México, Tlaxcala, Puebla, Guanajuato, Jalisco, Michoacán, Hidalgo, Querétaro y San Luis Potosí. Los cuales representan un total de 41907 colonias.
Con la finalidad de que los resultados del presente estudio pudieran ser comparables con los obtenidos en países donde se ha estimado la tasa de pérdida de colonias de abejas y se han identificado factores asociados a ésta, las preguntas de la encuesta fueron similares a las de los trabajos de Giray et al. (2010), Van Engelsdorp et al. (2010, 2011) y Van Der Zee et al. (2012), así como con lo señalado por la asociación internacional de investigadores sobre la prevención de pérdidas de colonias de abejas melíferas (Van Der Zee et al. 2013).

Las preguntas de la encuesta se dirigieron para obtener información sobre la ubicación y movilización de las colonias, promedio de producción de miel en el otoño de 2015 y número de colonias antes y después del invierno de 20152016. Además, se solicitó a los apicultores que indicaran las causas de pérdida de sus colonias. Con el propósito de identificar la presencia de los signos clínicos descritos del síndrome de colapso de comena (SCC) se les preguntó: ¿Cuántas colonias tenían la reina, alimento y muy poca población, ¿Cuántas de las colonias perdidas no tenían abejas muertas en la colmena?, ¿Cuántas de las colonias perdidas tenían cría muerta en las celdillas?, ¿Cuántas de las colonias perdidas tenían alimento en los panales? y la presencia de enfermedades y los métodos de control que utilizan.

\section{Cálculos y análisis estadísticos}

Se calculó la frecuencia (\%) de apicultores que movilizan colmenas a distancias menores a 200 km, de 201 a 500 km, de 501 a 1000 km y más de $1000 \mathrm{~km}$. El porcentaje de pérdida de colonias se calculó dividiendo el número total de colonias perdidas durante el invierno 2015-2016 entre el número total de colonias en riesgo en el mismo período de tiempo, multiplicado por 100 . Además, se calculó el intervalo de confianza al 95\% (IC 95\%) con un modelo lineal (SAS 2004).

Con la finalidad de determinar posibles diferencias estadísticas en la tasa de pérdida de colonias e las explotaciones apícolas de diferente tamaño, estas se clasificaron de acuerdo con Van Engelsdorp et al. (2010): hobby (1 a 50 colonias), 
actividad secundaria (51 a 500) y profesional (más de 500 colonias). La comparación de la pérdida de colonias entre regiones y entre el tamaño de explotaciones apícolas, se realizó por medio de la prueba de rangos Kruskal-Wallis. El análisis de los datos sobre la producción de miel entre estados y regiones se realizó con el análisis de varianza y la prueba $t$ de Student, respectivamente. Para determinar posibles diferencias entre las proporciones de apicultores que atribuyen la pérdida de sus colonias a factores como la enjambrazón, zanganeras, hambre, insecticidas, enfermedades, evasión, mal tiempo, mala calidad de las reinas y robo se utilizó la prueba $Q$ de Cochran, y la prueba logística de estimación de probabilidades binomiales (SAS 2004).

\section{RESULTADOS Y DISCUSIÓN}

Se estima que la tasa de pérdida de colonias fue del 33.4\% en el invierno de 2015-2016, sin diferencias significativas entre regiones de estudio $\left(\mathrm{X}^{2}=0.42, \mathrm{gl}=1, \mathrm{p}=0.51\right)$ (Tabla 1$)$. El $75 \%$ de los apicultores tuvieron una tasa de pérdida de colonias superior al $18 \%$. Estos resultados representan la primera estimación en dos regiones de México sobre la tasa de pérdida de colonias en el periodo invernal, la cual es superior a lo registrado en otros países (Van Engelsdorp et al. 2010). El porcentaje de pérdida de colonias del presente estudio es similar a los reportados por apicultores de los EUA (Van Engelsdorp et al. 2008, 2010, 2011, 2012, Spleen et al. 2013, Steinhauer et al. 2014, Lee et al. 2015) y al promedio de $12 \%$ de pérdida de colonias en países europeos (Brodschneider et al. 2016).

El $56 \%$ de los encuestados movilizan sus colmenas, el $46 \%$ a una distancia menor a $200 \mathrm{~km}$, el $17 \%$ de 201 a $500 \mathrm{~km}$, el $2.5 \%$ entre 501 y 1 $000 \mathrm{~km}$ y el $2 \%$ movilizan a más de $1,000 \mathrm{~km}$. El porcentaje de pérdida de colonias de los apicultores que no movilizan sus colonias fue de $31.2 \%$ (IC 95\%: $26.8-36.3 \%$ ) y para los apicultores que sí lo hacen fue del 34.6\%, (IC 95\%: 31.0-38.1\%). Pero no se observaron diferencias significativas entre ellos $\left(\mathrm{X}^{2}=1.18, \mathrm{gl}=1, \mathrm{p}=0.27\right)$. Tampoco se tuvieron diferencias significativas $\left(X^{2}=0.5, \mathrm{gl}=3\right.$, $p=0.08)$ en la tasa de pérdida de colonias entre apicultores que movilizan sus colonias a distancias menores a 200, 201 a 500, 501 a 1000 y más de 1000 $\mathrm{km}$. Estos resultados coinciden con los reportados por Oldroyd (2007) y Van Engelsdorp et al. (2010). Lo cual sugiere que el estrés provocado por la movilización de colonias no es un factor significativo que contribuya a las altas pérdidas de colonias.

Se ha reportado que el tamaño de la explotación apícola es un factor que se encuentra asociado a la tasa de pérdida de colonias (Chauzat et al. 2016), en las explotaciones profesionales es más probable que el manejo sea más intenso, las renten para polinización y exista un elevado hacinamiento de colonias, lo que contribuye a la transmisión de enfermedades, mientras que los apicultores de hobby tienden a ser sedentarios, tienen pocas colonias y las manejan menos rigurosamente. Los apicultores para los cuales la apicultura es una actividad económica secundaria tienden a ubicarse entre los dos grupos antes descritos (Royce y Rossignol 1990). En el presente estudio no se encontraron diferencias significativas $\left(X^{2}=1.29, p=0.52\right)$ en la tasa de pérdida de colonias entre apicultores de tipo hobby, actividad secundaria y profesionales (Tabla 2), por lo que el tamaño de las explotaciones apícolas en este estudio no fue un factor que influya en la pérdida de colonias, lo que coincide con lo reportado por Van Engelsdrop et al. (2010). Tampoco se observó efecto significativo $(p>0.05)$ de la movilización de colonias sobre la tasa de pérdida en explotaciones tipo hobby, secundaria y profesional, lo cual concuerda con lo reportado por Lee et al. (2015).

Se tuvieron diferencias significativas $(\mathrm{Q}=$ $135, p<0.0001$ ) en los factores que los apicultores encuestados consideran la causa de pérdida de sus colonias, la mayoría señaló múltiples razones, siendo respuestas más comunes el mal tiempo $(52.6 \%$ de los encuestados), seguido de las enfermedades y plagas $(42 \%)$, insecticidas (41\%), evasión (38\%), enjambrazón (28\%), colonias zanganeras (28\%), hambre $(20 \%)$, mala calidad de las reinas $(6 \%)$ y robo $(4 \%)$. Considerando las regiones y la frecuencia de apicultores que estiman la pérdida de sus colonias a los diversos factores, solo se observaron diferencias 
Tabla 1. Número de apicultores encuestados, número de colonias, pérdida promedio de colonias durante el invierno 2015-2016 e intervalo de confianza.

\begin{tabular}{ccccc}
\hline Región & Número de encuestados & Número de colonias & Pérdida promedio (\%) & Intervalo de confianza (95\%) \\
\hline Altiplano & 86 & 20,979 & 32.5 & $28.2-36.6$ \\
Norte & 110 & 20,928 & 34.3 & $30.3-38.4$ \\
Total & 196 & 41,907 & 33.4 & $30.6-36.4$ \\
\hline
\end{tabular}

Tabla 2. Número de apicultores encuestados, número de colonias antes del invierno 2015-2016, porcentaje promedio de pérdida de colonias de abejas melíferas e intervalo de confianza por tipo de explotación.

\begin{tabular}{ccccc}
\hline Tipo de explotación & $\begin{array}{c}\text { Apicultores } \\
\text { encuestados }\end{array}$ & $\begin{array}{c}\text { Número de colonias antes } \\
\text { del invierno 2015-2016 }\end{array}$ & $\begin{array}{c}\text { Media (\%) de } \\
\text { pérdida de colonias }\end{array}$ & $\begin{array}{c}\text { Intervalo de } \\
\text { confianza (95\%) }\end{array}$ \\
\hline Hobby & 59 & 1484 & 32.6 & $26.3-39.0$ \\
Actividad Secundaria & 117 & 17740 & 34.1 & $30.7-37.5$ \\
Profesional & 20 & 22683 & 32.6 & $22.6-42.6$ \\
\hline
\end{tabular}

Tabla 3. Frecuencia (\%) de apicultores que atribuyen la causa de pérdida de sus colonias a diversos factores

\begin{tabular}{lccc}
\hline \multicolumn{1}{c}{ Factor/Región } & Altiplano & Norte & $\mathrm{X}^{2}$ y p \\
\hline Enjambrazón & 32 & 28 & $0.26,0.60$ \\
Zanganeras & 30 & 27 & $0.21,0.64$ \\
Hambre & 23 & 20 & $0.21,0.64$ \\
Insecticidas & 41 & 42 & $0.03,0.85$ \\
Enfermedades & 46 & 39 & $0.79,0.31$ \\
Evasión & 32 & 42 & $2.02,0.15$ \\
Mal tiempo & 59 & 45 & $3.62,0.05$ \\
Mala calidad de la reina & 8 & 5 & $0.35,0.55$ \\
Robo & 10 & 0 & $11.6,0.0006$ \\
\hline
\end{tabular}

significativas respecto con el mal tiempo y el robo (Tabla 3). A excepción de los problemas derivados de la africanización de las abejas, los factores que los apicultores señalan coinciden con lo reportado por los apicultores de los EUA, quienes atribuyen sus pérdidas de colonias a la mala calidad de las reinas, $V$. destructor, inanición, debilidad de las colonias en otoño, pesticidas, malas condiciones de invernada y el SCC (Van Engelsdorp et al. 2010, 2011, 2012, Spleen et al. 2013, Steinhauer et al. 2014, Lee et al. 2015). También el genotipo de las abejas y el clima fueron los principales causantes de las altas pérdidas de colonias en Turquía (Giray et al. 2007). Mientras que en Europa, el tratamiento inadecuado contra la varroosis, loque americana, nosemosis, el acceso de las abejas a ciertos cultivos, la edad y los problemas de la reina en verano afectaron la mortalidad invernal de las colonias (Chauzat et al. 2010, Van Der Zee et al. 2014). Adicionalmente, en Canadá se encontró que el ácaro $V$. destructor se asocia al $85 \%$ de las muertes de colonias durante el invierno (Guzmán-Novoa et al. 2010). Se tuvieron diferen- cias significativas $(\mathrm{Q}=575.3, \mathrm{p}<0.0001)$ en la proporción de apicultores que reportan la presencia de enfermedades y plagas en sus colonias, el $87 \%$ de ellos reporta la presencia de varroosis, el $27 \%$ a la cría calcárea, $15 \%$ a loque americana, $15 \%$ loque europea, $11 \%$ nosemosis, $4 \%$ al pequeño escarabajo de la colmena (Aethina tumida) y $4 \%$ polilla de la cera (Galleria mellonella).

El $85 \%$ de los apicultores indicaron que aplican algún tratamiento contra $V$. destructor, al respecto se sabe que los acaricidas sintéticos pueden afectar el desarrollo de las reinas y zánganos, la habilidad de aprendizaje y la postura de la reina (Pettis et al. 2004). Además los ácidos orgánicos reducen la longevidad y sobrevivencia de obreras y la cría. Mientras que los monoterpenos constituyentes de los aceites esenciales derivados de plantas, tienen efectos tóxicos y suprimen la expresión genética relacionada con la inmunidad (Boncristiani et al. 2012). Al respecto se observó que el $29 \%$ de los apicultores utilizan acaricidas sintéticos (15\% de ellos aplica flumetrina, $8 \%$ fluvalinato y $6 \%$ amitraz), el $33 \%$ 
utiliza ácidos orgánicos (30\% ácido oxálico y 3\% ácido fórmico) y el $56 \%$ aplica aceites esenciales ( $42 \%$ timol y $14 \%$ diversas preparaciones caseras). El hecho de que los apicultores utilicen estos productos para el control de $V$. destructor puede estar contribuyendo a la pérdida de colonias.

El promedio de producción de miel que reportan los apicultores fue de $13.5 \pm 0.97 \mathrm{~kg}$, con variación de 0 a $75 \mathrm{~kg}$, no hubo diferencias significativas en la producción de miel entre estados, ni regiones $(\mathrm{F}=1.13, \mathrm{p}=0.33 ; t=1.01, \mathrm{p}=0.31$, respectivamente), lo que posiblemente se deba a la variación de condiciones de manejo, genética de las abejas y condiciones ambientales entre las regiones. Del total de colonias perdidas, el $61 \%$ tenían reina, alimento y muy poca población, el $43 \%$ no tenían abejas muertas en las colmenas, $13 \%$ tenían cría muerta en las celdillas y el $60 \%$ tenían alimento en los panales, estos signos sugieren la presencia del SCC, sin embargo, es necesaria la realización de ex- perimentos controlados que permitan llegar a resultados concluyentes.

La tasa promedio estimada de pérdida de colonias en las regiones del altiplano y norte de México fue del $33.4 \%$, sin diferencias entre las regiones. La pérdida de colonias fue atribuida al mal tiempo, enfermedades y plagas, insecticidas y problemas derivados de la africanización de las abejas y del manejo. Considerando que muchos de los factores clasificados como las principales causas de pérdida de las colonias pueden estar relacionados con el manejo, se sugiere mejorar las condiciones de sus colonias con suplementación alimenticia, uso de equipo adecuado, monitoreo del nivel de infestación de $V$. destructor, control integral de enfermedades, uso adecuado de medicamentos autorizados, remplazo de panales viejos, desinfección de equipo, unión de colonias débiles, remplazo de reinas y uso de genotipos resistentes a $V$. destructor y otras enfermedades.

\section{LITERATURA CITADA}

Boncristiani H, Underwood R, Schwarz R, Evans JD, Pettis J (2012) Direct effect of acaricides on pathogen loads and gene expression levels in honey bees Apis mellifera. Journal of Insect Physiology 58: 613-620.

Brodschneider R, Gray A, Van Der Zee, R, Adjlane N, Brusbardis V, Charrière JD, et al. (2016) Preliminary analysis of loss rates of honey bee colonies during winter 2015/16 from the COLOSS survey. Journal of Apicultural Research 55: 375-378.

CGG (2010) Situación actual y perspectiva de la apicultura en México. Coordinación General de Ganadería SAGARPA. Claridades Agropecuarias 199: 3-34. http://www.infoaserca.gob.mx/claridades/revistas/199 /ca199-3.pdf. Fecha de consulta 15 de abril de 2017.

Chauzat MP, Jacques A. Laurent M, Bougeard S, Hendrikx P, Ribière-Chabert, et al. (2016) Risk indicators affecting honeybee colony survival in Europe: one year of surveillance. Apidologie 47: 348-378.

Chauzat MP, Martel AC, Zeggane S, Drajnudel P, Schurr F, Clément, MC, et al. (2010) A case control study and a survey on mortalities of honey bee colonies (Apis mellifera) in France during the winter of 2005-6. Journal of Apicultural Research 49: 40-51.

Giray T, Cakmak I, Aydin L, Kandemir I, Inci A, Oskay D, et al. (2007) Preliminary survey results on 2006-2007 colony losses in Turkey. Uludag Bee Journal 7: 101-107.

Giray T, Kence M, Oskay D, Döke MA, Kence A (2010) Scientific note: colony losses survey in Turkey and causes of bee deaths. Apidologie 41: 451-453.

Guzmán-Novoa E, Eccles L, Calvete Y, Mcgowan J, Kelly PG, Correa-Benítez A (2010) Varroa destructor is the main culprit for the death and reduced populations of overwintered honey bee (Apis mellifera) colonies in Ontario, Canada. Apidologie 41: 443-450. 
Guzmán-Novoa E (2016) Colony collapse disorder and other threats to honey bees. In: Cork S, Hall D, Liljebjelke $\mathrm{K}$ (ed) One health case studies: Addressing complex problems in a changing World. $5 \mathrm{~m}$ Publishing, 1 edition. UK. pp: 205-216.

Lee KV, Steinhauer N, Rennich K, Wilson ME, Tarpy DR, Caron DM, et al (2015) A national survey of managed honey bee 2013-2014 annual colony losses in the USA. Apidologie 46: 292-305.

Neumann P, Carreck NL (2010) Honey bee colony losses. Journal of Apicultural Research 49: 1-6.

Oldroyd PB (2007) What's killing American honey bees? Plos Biology 5:168.

Pettis JS, Collins A, Wilbanks R (2004) Effects of coumaphos on queen rearing in the honey bee, Apis mellifera. Apidologie 35: 605-610.

Royce LA, Rossignol PA (1990) Honey bee mortality due to tracheal mite parasitism. Parasitology 100 : 147-151.

SAS (2004) SAS/STAT ${ }^{\circledR} 9.1$ user's guide. SAS Publishing. SAS Institute Inc., NC, USA. 5121p.

Spleen AM, Lengerich EJ, Rennich K, Caron D, Rose R, Pettis JS, et al. (2013) A national survey of managed honey bee 2011-12 winter colony losses in the United States: results from the Bee Informed Partnership. Journal of Apicultural Research 52: 44-53.

Stankus T (2014) Reviews of science for science librarians: an update on honeybee colony collapse disorder. Science and Technology Libraries 33: 228-260.

Steinhauer NA, Rennich K, Wilson ME, Caron DM, Lengerich EJ, Pettis JS, et al. (2014) A national survey of managed honey bee 2012-2013 annual colony losses in the USA: results from the Bee Informed Partnership. Journal of Apicultural Research 53: 1-18.

Van Der Zee R, Pisa L, Andonov S, Brodschneider R, Charriere JD, Chlebo R, et al. (2012) Managed honey bee colony losses in Canada, China, Europe, Israel and Turkey, for the winters of 2008-9 and 2009-10. Journal of Apicultural Research 51: 100-114.

Van Der Zee R, Gray A, Holzmann C, Pisa L, Brodschneider R, Chlebo R, et al. (2013) Standard survey methods for estimating colony losses and explanatory risk factors in Apis mellifera. Journal of Apicultural Research 52: 1-36.

Van Der Zee R, Brodschneider R, Brusbardis V, Charriere JD, Chlebo R, Coffey MF, et al. (2014) Results of international standardised beekeeper surveys of colony losses for winter 2012-2013: analysis of winter loss rates and mixed effects modelling of risk factors for winter loss. Journal of Apicultural Research 53: 19-34.

Van Engelsdorp D, Hayes JJ, Underwood RM, Pettis J (2008) A survey of honey bee colony losses in the U.S. Fall 2007 to Spring 2008. PLoS One 3:4071.

Van Engelsdorp D, Evans JD, Saegerman C, Mullin C, Haubruge E, Nguyen BK, et al. (2009) Colony collapse disorder: a descriptive study. PloS One 4:6481.

Van Engelsdorp D, Hayes JJ, Underwood RM, Pettis JS (2010) A survey of honey bee colony losses in the United States, fall 2008 to spring 2009. Journal of apicultural research 49: 7-14.

Van Engelsdorp D, Hayes JJ, Underwood RM, Caron D, Pettis J (2011) A survey of managed honey bee colony losses in the USA, fall 2009 to winter 2010. Journal of Apicultural Research 50: 1-10. 
Van Engelsdorp D, Caron D, Hayes J, Underwood R, Henson, M; Rennich K, et al. (2012) A national survey of managed honey bee 2010-11 winter colony losses in the USA: results from the Bee Informed Partnership. Journal of Apicultural Research 51: 115-124. 
